\title{
Protocolo interinstitucional de abordaje foniátrico de la disfagia orofaríngea en pacientes con COVID-19
}

\section{Interinstitutional protocol for phoniatric approach to oropharyngeal dysphagia in patients with COVID-19}

\author{
Mónica Segura-Hernández, * Víctor Manuel Valadez-Jiménez, ${ }^{\ddagger}$ \\ Daniela Carolina Tejeda-Franco, ${ }^{\S}$ Annel Gómez-Coello ${ }^{\ddagger}$
}

Citar como: Segura-Hernández M, Valadez-Jiménez VM, Tejeda-Franco DC, Gómez-Coello A. Protocolo interinstitucional de abordaje foniátrico de la disfagia orofaríngea en pacientes con COVID-19. An Med (Mex). 2021; 66 (1): 42-47. https://dx.doi.org/10.35366/99488

\section{RESUMEN}

El SARS-CoV-2 es un coronavirus B, denominado así por la apariencia que da bajo el microscopio electrónico parecido a una corona. El 30 de enero de 2020, la Organización Mundial de la Salud declaró oficialmente la epidemia COVID-19 como una emergencia de salud pública de preocupación internacional. El manejo clínico de un paciente con enfermedad por SARSCoV-2 en la unidad de cuidados intensivos y de todas las comorbilidades que esto implica, es de gran importancia para el médico foniatra, el cual será esencial en el diagnóstico y la rehabilitación de la disfagia orofaríngea de los pacientes. Este trabajo en particular presta especial atención al protocolo de diagnóstico y manejo rehabilitatorio de la disfagia orofaríngea en pacientes que cursaron con esta enfermedad.

Palabras clave: Coronavirus, foniatría, disfagia.

Nivel de evidencia: III

\begin{abstract}
SARS-CoV-2 is a coronavirus type B, named because of its crown-like appearance under the electron microscope. On January 30, 2020, The World Health Organization officially declared the COVID-19 epidemic a public health emergency of international concern. The clinical management of a patient with coronavirus disease in the Intensive Care Unit and of all the comorbidities that this implies, is of great importance for the phoniatric physician, which will be essential in the diagnosis and rehabilitation of oropharyngeal dysphagia in patients. This work in particular pays special attention to the protocol for the diagnosis and rehanilitation management of oropharyngeal dysphagia in patients with this disease.
\end{abstract}

Keywords: Coronavirus, phoniatrics, dysphagia.

Level of evidence: III
* Centro Médico American British Cowdray / Hospital General de México.

₹ Jefe de la División de Foniatría. Instituto Nacional de Rehabilitación.

$\S$ Centro Médico Nacional 20 de Noviembre.

Especialidad en Audiología, Otoneurología y Foniatría. Alta Especialidad en Métodos Especiales de Diagnóstico y Tratamiento en Foniatría.

Ciudad de México.
Recibido: 07/12/2020. Aceptado: 28/03/2021.

Correspondencia: Dra. Annel Gómez Coello Dr. Balmis Núm. 148,

Col. Doctores, 06720

Alcaldía Cuauhtémoc, Ciudad de México.

Tel: 55 1879-2000, ext. 6311.

E-mail: annelgomezc@gmail.com 


\section{INTRODUCCIÓN}

Hasta el 15 de septiembre de 2020 la OMS ha reportado más de 29.8 millones de casos y casi un millón de muertes en todo el mundo, derivados de la infección por el virus $S A R S-C o V-2$ originada en diciembre de 2019 en la región de Wuhan, China. Actualmente las investigaciones científicas se centran en el mejor conocimiento de la infección aguda y de sus estrategias terapéuticas. ${ }^{1}$ Sin embargo, aún no se ha dimensionado la magnitud de las secuelas y la importancia de su pronto abordaje para disminuir el riesgo de complicaciones. Este trabajo en particular presta especial atención al diagnóstico y manejo rehabilitatorio de la disfagia orofaríngea en pacientes que cursaron con esta enfermedad.

\section{Generalidades}

Los coronavirus son virus envueltos de ARN de sentido positivo no segmentados que pertenecen a la familia Coronaviridae y al orden nidovirales, y se transmiten con facilidad en humanos y otros mamíferos. ${ }^{2}$ Los coronavirus se replican primordialmente en las células epiteliales del tracto respiratorio inferior y en menor medida en el tracto respiratorio superior. ${ }^{3}$ Después de los contagios en casa y el trabajo, los brotes nosocomiales representan aproximadamente un tercio de las formas de transmisión a nivel mundial, relacionado a que el virus es más estable a temperaturas inferiores $\left(20^{\circ}\right)$ y bajas condiciones de humedad, presente en los hospitales o consultorios con mayor permanencia en superficies de plástico o acero. Estas propiedades favorecen su permanencia en instalaciones médicas equipadas y sometidas a aire acondicionado, además de un alto riesgo de súper propagación por los procedimientos generadores de aerosoles (AGP) como el uso continuo de presión positiva en las vías respiratorias, nebulización de medicamentos, reanimación cardiopulmonar, intubación, nasoendoscopia, evaluación clínica e instrumentada de la deglución.

En referencia a nuestro artículo, el abordaje foniátrico puede considerarse como de alto riesgo de contagio, ya que tanto la exploración clínica como instrumentada son consideradas AGP, por lo que dicho abordaje debe ser postergado el mayor tiempo posible. ${ }^{4,5}$ Por otra parte, la rápida propagación se atribuyó al mal cumplimiento de los trabajadores de la salud con el uso de equipo de protección y los escasos protocolos de sanidad durante los procedimientos. ${ }^{4}$

La enfermedad por coronavirus 2019 (COVID-19) puede ser asintomática; sin embargo, de 14 a $20 \%$ pueden complicarse con una neumonía de infiltrados bilaterales y en $5 \%$ pueden presentar síndrome de distrés respiratorio agudo (SDRA), ${ }^{6}$ coagulopatía intravascular diseminada, edema laríngeo y laringitis en el paciente crítico que puede ocasionar obstrucción de la vía aérea o disfagia orofaríngea, neumonía necrosante por sobreinfección, complicaciones cardiovasculares, sepsis, choque séptico, embolia pulmonar masiva y fallo multiorgánico. ${ }^{7}$

\section{Disfagia orofaríngea en los pacientes con COVID-19}

Por tratarse de pacientes en estado crítico, la disfagia orofaríngea está relacionada con la intubación endotraqueal y con la duración de la ventilación mecánica; sin embargo, es también atribuida a cambios multifactoriales principalmente mecánicos y cognitivos, entre los que se encuentran: debilidad muscular, traumatismo orofaríngeo y laríngeo, sarcopenia, alteraciones sensoriomotoras, sobre todo cuando el paciente se encuentra en estado de delirio, secundario al efecto residual de la medicación narcótica y ansiolítica. ${ }^{8}$

Debido a que estos pacientes presentan un alto riesgo de aspiración de los alimentos y de las propias secreciones, el abordaje foniátrico es imprescindible para la toma de decisiones sobre la ruta de alimentación más segura, por lo que a continuación ahondaremos en las posibles pruebas clínicas y diagnósticas a realizar en estos pacientes, siempre considerando el estricto apego a las medidas de protección.

\section{Postura actual sobre el abordaje foniátrico}

La Sociedad de Investigación de la Disfagia estipula que los siguientes procedimientos deben considerarse como AGP y por tanto, requieren que se tomen todas las precauciones como el uso adecuado del equipo de protección así como del ambiente donde se llevarán a cabo mientras se realizan. Estos procedimientos incluyen cualquier examen realizado en el tracto aerodigestivo (oído medio, nariz, faringe, cavidad oral, orofaringe, hipofaringe o esófago) como: ${ }^{9,10}$

1. Evaluación clínica de la deglución que incluye: examen oromotor y de pares craneales, prueba del reflejo tusígeno y estado bucal.

2. Pruebas clínicas de apoyo para la evaluación de la disfagia:

a. El test del agua, desarrollado y validado por DePippo, con una sensibilidad de $84.6 \%$ y un especificidad de $75 \%$ para la predicción clínica para aspiración. 
b. El método de exploración clínica volumen viscosidad (MECV-V), útil en el tamizaje de aspiraciones, cuenta con una sensibilidad diagnóstica de 83 a $85 \%$ y especificidad de $63 \%$.

c. La ausculta cervical, complementaria a las dos primeras pruebas, presenta $66 \%$ de especificidad y $62 \%$ de sensibilidad (con respecto a la aspiración-penetración).

d. La evaluación fibroendoscópica de la deglución (FEES, Fiberoptic Evaluation of Swallowing), en la que se ha reportado $88 \%$ de sensibilidad y $90 \%$ de concordancia con la videofluoroscopia respecto de la presencia de aspiración a la vía aérea.

3. Estudio videofluoroscópico de la deglución (VFSS).

4. Cuidado y manejo de la laringectomía, que incluyen: restauración quirúrgica de la voz (cambio de prótesis e inspección del estoma).

5. Intubación, cuidado y manejo de traqueostomía, con o sin ventilación mecánica: procedimientos de succión, desinflado del manguito, oclusión digital, uso de válvulas del habla.

6. Ventilación no invasiva y soporte respiratorio de alto flujo mediante cánulas nasales, cánulas de alto flujo y mascarilla.

7. Entrenamiento de la fuerza de los músculos respiratorios.

8. Manometría faríngea-esofágica.

En marzo de 2020, la British Laryngological Association (BLA) y la British Association of Otorhinolaryngology-Head and Neck (ENT-UK) emitieron declaraciones con respecto a la nasoendoscopia y a la FEES, que incluían la restricción del estudio en pacientes infectados; sin embargo, algunos pacientes ameritarán la evaluación, y esta decisión siempre debe ser multidisciplinaria, discutida y planificada formalmente por el equipo de trabajo antes de realizar el procedimiento, considerando los riesgos y beneficios. Es por ello que la FEES sólo debe realizarse si es absolutamente necesario como en los casos en que se sospeche de neumonía agregada por broncoaspiración, aspiraciones silentes u obstrucción de la vía aérea para informar al equipo médico sobre el manejo inmediato del paciente..$^{5,11}$

\section{Protocolo interinstitucional de abordaje foniátrico}

Este protocolo es realizado en el Centro Médico American British Cowdray, el Hospital General de México Dr. Eduardo Liceaga, Centro Médico Nacion- al 20 de Noviembre del ISSSTE y el Instituto Nacional de Rehabilitación «Dr. Luis Guillermo Ibarra Ibarra», y es resultado del consenso de los servicios de foniatría, basado en las guías internacionales y en la experiencia de dichos servicios durante la atención en la pandemia por coronavirus.

\section{Toma de decisiones clínicas para el abordaje foniátrico}

\section{A) Estatus infeccioso del paciente}

En el contexto actual, teóricamente todos los pacientes se consideran SARS-CoV-2 positivos. Sin embargo, se puede aplicar la siguiente categoría:

1. Pacientes positivos para $S A R S-C o V-2$ : son los pacientes que cuenten con una prueba de PCR positiva, y/o una tomografía computarizada de tórax sugestiva de lesiones por SARS-CoV-2.

2. Pacientes sospechosos cuando: existan signos clínicos sugestivos como mialgia, astenia, fiebre, tos, dolor de pecho, disnea, dolor de cabeza, anosmia o ageusia (pérdida del gusto u olfato), síntomas digestivos (especialmente diarrea), delirio, o que hayan estado en contacto con una persona contagiada. ${ }^{11}$

\section{B) Tiempo estimado para la evaluación foniátrica}

Aún no se han realizado suficientes estudios aleatorizados para determinar el tiempo exacto para la realización y la valoración foniátrica de los pacientes con COVID-19, todo dependerá de los criterios de urgencia y necesidades del paciente; sin embargo, se aconseja que se realice una vez que el paciente presente evidencia documentada de dos muestras virológicas consecutivas negativas (es decir, hisopado nasofaríngeo) con 24 horas de diferencia, además de tener como requisito que el médico utilice el equipo de protección personal adecuado. ${ }^{12,13}$ Esto en ocasiones no es posible en servicios como el de nuestras instituciones, por lo que hemos realizado dichas valoraciones en pacientes hospitalizados, e inclusive durante su estancia en las unidades de cuidados intensivos, ya que la nasolaringoendoscopia y/o la FEES llega a ser determinante para la toma de decisiones y evolución del paciente.

\section{C) Criterios para realizar las pruebas de deglución}

De acuerdo con la guía realizada por el Real Colegio de Terapeutas de Habla y de Lenguaje (RCSLT), 
respaldada por las asociaciones: Asociación Británica de Laringología, el Consejo de la Sociedad Torácica Británica, la ENT-UK y los miembros del Proyecto Nacional de la Traqueotomía se consideran criterios específicos para llevar a cabo la evaluación foniátrica en estos pacientes, graduados en tres niveles (Figura 1). ${ }^{14}$

\section{Consideraciones óptimas para realizar la prueba}

\section{A) Uso indispensable y forzoso de equipo de protección personal (EPP)}

Los profesionales de la salud estamos obligados a estar informados sobre el COVID-19, el riesgo de transmisión y el uso apropiado del EPP. Cuando se atiende a un paciente sospechoso o confirmado, el EPP recomendado incluye un respirador de aire motorizado (PAPR) o la mascarilla N95, gafas o careta desechable para protección ocular, guantes y bata de aislamiento. Todos los profesionales de la salud deben recibir capacitación y demostrar competencia para colocarse, retirarse y desechar o desinfectar adecuadamente el equipo de protección personal. ${ }^{15}$ Es necesario no realizar un procedimiento generado de aerosoles como la nasolaringoendoscopia, si no se dispone de un equipo de protección personal adecuado.

\section{B) Paciente}

Antes de realizar una FEES o nasolaringoendoscopia, por tratarse de pacientes en estado crítico o bien postinfección por $S A R S-C o V-2$, tenemos que asegurarnos de que las condiciones clínicas del paciente sean óptimas para la realización de la prueba considerando lo siguiente: ${ }^{16}$

1. Estado neurológico: si el paciente presenta deliro, sedación, patología neurológica agregada.

2. Estado hemodinámico: si aún es dependiente de vasopresores, estabilidad de frecuencia cardiaca y presión arterial.

3. Estado respiratorio: si el paciente es dependiente de ventilador, si está o estuvo con intubación endotraqueal y tiempo de la misma, si tiene traqueostomía, si se encuentra taquipneico o dependiente de oxígeno (ya sea en alto flujo, CPAP o puntas nasales).

4. Estado hemato-infeccioso: el paciente debe encontrarse afebril o sin necesidad de antipiréticos,

1. Sospecha de alteraciones de las estructuras laríngeas que conduzcan al compromiso de las vías respiratorias

2. Informar el destete y la decanulación

3. Toma de decisiones con respecto a la alimentación oral cuando no existe un método alternativo de nutrición y hay sospecha de aspiración silente

4. Informar la decisión inmediata al equipo multidisciplinario para determinar el alta hospitalaria

1. La evaluación puede programarse y retrasarse sin afectar negativamente los resultados o la seguridad del paciente
1. Por el estado general del paciente se podrían utilizar otras modalidades de evaluación clínica (método de exploración clínica, volumen, viscosidad, ausculta cervical, test de agua, etcétera)

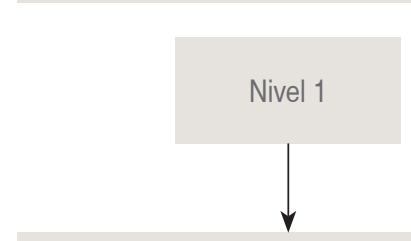

Criterios urgentes y esenciales que por la necesidad de toma de decisiones se requiere apoyarse en la evaluación clínica e instrumentada como la nasoesdoscopia o FEES

Figura 1: Criterios específicos para realizar la evaluación foniátrica. 
además considerar el riesgo de sangrado por uso de anticoagulantes.

5. Estado gastrointestinal: tipo de alimentación actual, presencia de sonda nasogástrica, nasoyeyunal, orogástrica o alimentación parenteral.

Si el paciente presenta alteración en alguno de los apartados antes mencionados, se debe postergar la prueba hasta que el paciente se encuentre en un estado general estable. En caso de no ser posible postergarlo, se recomienda siempre tomar en cuenta todas las variables al momento de llevar a cabo la valoración, y cómo éstas pueden influir en los resultados de la prueba.

\section{C) Médico}

El médico foniatra deberá tener las habilidades y la experiencia para tratar de minimizar el contacto con la mucosa nasal, faríngea y laríngea para reducir el riesgo de tos inducida y estimulación del reflejo nauseoso así como la inserción, manipulación y extracción rápidas y precisas del nasendoscopio para obtener imágenes de la más alta calidad en el menor tiempo posible, además de asegurarse de que éste se retire con cuidado para minimizar las salpicaduras y limitar la exposición de los aerosoles. ${ }^{17}$

\section{D) Lugar de atención}

La evaluación debe realizarse en una habitación bien ventilada o en hospitalización con la menor cantidad de personal y mobiliario posible durante la prueba, desinfectando inmediatamente después de cada laringoscopia, nasoendoscopia y/o FEES. No se han establecido virucidas específicos, pero se recomienda la desinfección con peróxido de hidrógeno al $2 \%$, cloro de 2 a $5 \mathrm{~g} / \mathrm{L}$ o alcohol al $75 \% .{ }^{17,18}$

\section{Características de la prueba}

La evaluación fibroendoscópica de la deglución debe tener ciertas adaptaciones para optimizar su realización y reducir al mínimo el riesgo de contagio, utilizando el siguiente protocolo adaptado de Langmore: ${ }^{18}$

1. La prueba debe realizarse de manera eficiente para minimizar de exposición.

2. Los puntos importantes a evaluar y a reportar durante la prueba son:

a. Estructuras de laringe e hipofaringe en reposo. b. Cantidad, ubicación y respuesta fisiológica a las secreciones (capacidad tusígena y deglutoria).

c. Fonación (según corresponda).

En la prueba se evalúan la consistencia de papillas, consistencia tipo miel, sólidos suaves, líquidos, jugos tipo néctar, con apoyo de una cuchara cafetera. $\mathrm{Y}$ se sugiere que exclusivamente se realicen dos evaluaciones en cantidades de media y una cucharada cafetera, o según la tolerancia del paciente, pero sin exceder de dos ensayos por consistencia, ya que el procedimiento debe ser lo más breve posible. Es importante considerar el estado actual del paciente para saber qué pruebas elegir para realizar la FEES.

Al concluir el procedimiento, se procederá a la desinfección y esterilización del nasoendoscopio mediante la inmersión en soluciones antisépticas durante aproximadamente 15 minutos, y a pesar de que no se han establecido las fórmulas óptimas de esterilización, se ha reportado que la esterilización por gas con óxido de etileno y el reprocesamiento químico con alcohol isopropílico, glutaraldehído, dióxido de cloro u ortoftalaldehído (OPA) tienen un buen efecto virucida. ${ }^{14,18}$

\section{Resultados de la prueba}

Al finalizar el procedimiento, los hallazgos y recomendaciones deben discutirse con el equipo multidisciplinario y determinar el plan de manejo al paciente. Se recomienda que el informe incluya las siguientes escalas en el reporte de los resultados:

1. Escala de secreciones de Nueva Zelanda: especificando la localización, el porcentaje, la significancia, la facilidad o imposibilidad de aclaración de las secreciones. ${ }^{19}$

2. Escala de residuos de Yale: es una herramienta confiable, validada, definida anatómicamente y basada en imágenes para determinar la ubicación y la gravedad de los residuos en la prueba de deglución, que nos ayuda a clasificar los patrones de severidad de residuos en vallécula y senos piriformes, en nivel leve, moderado o severo, para propósitos de diagnóstico y toma de decisiones terapéuticas. $^{20}$

3. Escala de aspiración/penetración de Rosenbek, adaptada por Colodny para la FEES: describe si el material: a. No ingresa a las vías respiratorias. b. Ingresa a la laringe, pero permanece por encima de las cuerdas vocales. c. Ingresa a la laringe hasta el nivel de las cuerdas vocales, o d. Pasa por debajo de las cuerdas vocales. ${ }^{21,22}$ 
Además del reporte claro de los hallazgos en el estudio, se debe indicar de forma precisa la ruta de alimentación más segura para el paciente así como esclarecer las medidas de deglución segura ambientales, posturales, de cantidad y consistencias. Las imágenes de preferencia deberán ser grabadas para su revisión conjunta y buscar la opinión de otras áreas como terapia de lenguaje y deglución para facilitar el consenso sobre la gestión y el seguimiento del tratamiento. Todo esto con la finalidad de evitar la repetición innecesaria del procedimiento.

\section{CONCLUSIÓN}

La valoración foniátrica en pacientes con infección por el SARS-CoV-2 puede aportar beneficios en la toma de decisiones y en el manejo de los pacientes con disfagia orofaríngea, aunque aún exista falta de consenso acerca del momento idóneo de su realización; se recomienda que dicha valoración se lleve a cabo sólo si es un criterio urgente o necesario para la toma de decisiones, lo ideal es realizarla una vez que el paciente presente pruebas serológicas negativas bajo todas las especificaciones señaladas previamente. Sin embargo, a pesar de que el abordaje foniátrico se considera un procedimiento de alto riesgo para el personal médico, puede realizarse y reducirse drásticamente el riesgo de contraer la infección con el cumplimiento estricto de las medidas de protección y recomendaciones técnicas descritas.

\section{REFERENCIAS}

1. Orientaciones técnicas sobre el nuevo coronavirus (2019nCoV). Organización Mundial de la Salud. 2020. Disponible en: https://www.who.int/es/emergencies/diseases/novelcoronavirus-2019/technical-guidance.

2. Palacios CM, Santos E, Velázquez CM, León JM. COVID-19, a worldwide public health emergency. Rev Clin Esp. 2021; 221 (1): 55-61.

3. Perlman S. Another decade, another coronavirus. N Engl J Med. 2020; 382 (8): 760-762.

4. Hui DS, Azhar EI, Kim YJ, Memish ZA, Oh M don, Zumla A. Middle East respiratory syndrome coronavirus: risk factors and determinants of primary, household, and nosocomial transmission. Lancet Infect Dis. 2018; 18 (8): 217-227.

5. Davies E, Roland N. Nasal endoscopy and laryngoscopy examination of ENT patients. (March 23, 2020). Available from: https://www.entuk.org/nasal-endoscopy-andlaryngoscopy-examination-ent-patients.

6. Cinesi GC, Peñuelas RO, Luján TM, Egea SC, Masa JJ, García FJ et al. Clinical consensus recommendations regarding noninvasive respiratory support in the adult patient with acute respiratory failure secondary to SARS-CoV-2 infection. Arch Bronconeumol. 2020; 56 (Suppl 2): 11-18.

7. Azer SA. COVID-19: pathophysiology, diagnosis, complications and investigational therapeutics. New Microbes New Infect. 2020; 37: 100738.

8. Frajkova Z, Tedla M, Tedlova E, Suchankova M, Geneid A. Postintubation dysphagia during COVID-19 outbreakcontemporary review. Dysphagia. 2020; 35 (4): 549-557.

9. Clavé P, Arreola V, Velasco M, Quer M, Castellví JM, Almirall $\mathrm{J}$ et al. Diagnóstico y tratamiento de la disfagia orofaríngea funcional. Aspectos de interés para el cirujano digestivo. Cir Esp. 2007; 82 (2): 62-76.

10. Rubio-Grayeb ML, Villeda-Miranda A, Arch-Tirado E, Martínez-Wbaldo MC. Concordancia entre fibroendoscopia y auscultación cervical en la disfagia de sujetos con enfermedad de Parkinson. Rev Mex AMCAOF. 2016; 5 (3): 83-88.

11. Updated and Amended message from the BLA President. Mark Watson. March 21, 2020. 2020. Available from: https:/ www.britishlaryngological.org/news/updated-and-amendedmessage-bla-president-mark-watson.

12. Mattei A, Amy de la Bretèque B, Crestani S, Crevier-Buchman L, Galant C, Hans S. Guidelines of clinical practice for the management of swallowing disorders and recent dysphonia in the context of the COVID-19 pandemic. Eur Ann Otorhinolaryngol Head Neck Dis. 2020; 137 (3): 173-175.

13. Bolton L, Mills C, Wallace S, Brady MC. Aerosol generating procedures, dysphagia assessment and COVID-19: a rapid review. Int J Lang Commun Disord. 2020; 55 (4): 629-636.

14. Simpson R, Robinson L. Rehabilitation after critical illness in people with COVID-19 infection. Am J Phys Med Rehabil. 2020; 99 (6): 470-474.

15. Speech and language therapist-led endoscopic procedures in the COVID-19 pandemic. $\mathrm{R}$ Coll Speech and Language. 2020; 1-17. Available from: https://www.rcslt.org/-/media/ docs/Covid/RCSLT-COVID-19-SLT-led-endoscopic-procedureguidance.

16. Modes of transmission of virus causing COVID-19: implications for IPC precaution recommendations, Geneva: World Health Organization; 2020. Available from: https:// www.who.int/publications-detail/modes-of-transmissionof-virus-causing-covid-19-implications-for-ipc-precautionrecommendations.

17. Miles A, Connor NP, Desai RV, Jadcherla S, Allen J, Brodsky M et al. Dysphagia care across the continuum: a multidisciplinary dysphagia research society taskforce report of service-delivery during the COVID-19 global pandemic. Dysphagia. 2021; 36 (2): $170-182$.

18. Rameau A, Young VN, Amin MR, Sulica L. Flexible Laryngoscopy and COVID-19. Otolaryngol Head Neck Surg. $2020 ; 162$ (6): 813-815.

19. Miles A, Hunting A, McFarlane M, Caddy D, Scott S. Predictive value of the New Zealand Secretion Scale (NZSS) for pneumonia. Dysphagia. 2018; 33 (1): 115-122.

20. Neubauer PD, Rademaker AW, Leder SB. The yale pharyngeal residue severity rating scale: an anatomically defined and image-based tool. Dysphagia. 2015; 30 (5): 521-528.

21. Rosenbek JC, Robbins JA, Roecker EB, Coyle JL, Wood JL. A penetration-aspiration scale. Dysphagia. 1996; 11 (2): 93-98.

22. Nazar MG, Ortega TA, Godoy MA, Godoy MJM, Fuentealba MI. Evaluación fibroscópica de la deglución. Rev Otorrinolaringol Cir Cabeza Cuello. 2008; 68 (2): 131-142. 